\title{
Simulation to Establish Correlation between PCA and Reactor Power Using Neutron Detector Signals
}

\author{
M. Sivaramakrishna
}

\begin{abstract}
-the classical nuclear instrumentation in fast reactors monitors the core neutron flux from shut down to full power over $\sim 10$ decades. This wide range necessitates combination of various detectors and electronic systems, with interlocks and overlapping. Also there is a tradeoff between the accuracy and response times. To overcome this problem, a special technique, viz. principal component analysis in frequency domain is proposed. This paper discusses the detailing of the technique, results obtained, future scope.
\end{abstract}

Index Terms-Neutron, pulse, counting, pica, correlation, vector

\section{INTRODUCTION}

In fast reactor, nuclear chain reactions are initiated, controlled, and sustained using fast neutrons, at a steady rate.

\section{A. Nuclear Instrumentation System}

It is imperative for an operator to know the amount of neutron power that is being generated so that he does not exceed the set power limit. Neutron Power level is capable of spanning about 10 orders of magnitude from the lowest power level to full power. In many nuclear power plants, 3 levels and methods of detection are used. These levels are called:

- Source Range (usually a proportional detector or fission counter)

- Intermediate Range (usually a compensated ion chamber)

- Power Range (usually an uncompensated ion chamber

The detector ranges overlap to ensure accuracy of the indication. This system also provides signals to the Reactor Protection System that can initiate automatic shutdown of the reactor,

\section{NEUTRON DETECTORS}

Unlike any other charge particles, neutrons being charge less can't be detected using ionization principle. Hence various neutron detectors have been developed in the past years based on the following principles to created charge in some nuclear reaction. These effectively overcome the challenges mentioned earlier. However, each has its own set of advantages and disadvantages. The main reactions used in counting neutrons are

$$
\begin{aligned}
& \bullet{ }_{0} \mathrm{n}^{1}+5 \mathrm{~B} 10 \rightarrow{ }_{2} \mathrm{He}^{4}+{ }_{3} \mathrm{Li}^{7} \\
& \bullet{ }_{0} \mathrm{n}^{1}+{ }_{2} \mathrm{He}^{3} \rightarrow{ }_{1} \mathrm{H}^{1}+{ }_{2} \mathrm{Li}^{3} \\
& \bullet{ }_{0} \mathrm{n}^{1}+92 \mathrm{U} 235 \rightarrow\left({ }_{\mathrm{N}} \mathrm{FP} 1^{\mathrm{M}}\right)^{*}+\left({ }_{92}-{ }_{\mathrm{N}} \mathrm{FP} 2^{235-\mathrm{M}-}\right. \\
& \left.{ }_{2}^{2}\right)^{*}+2{ }_{0} \mathrm{n}^{1}+\gamma
\end{aligned}
$$

Manuscript received December 7, 2010; revised July 6, 2011.

M. Sivaramakrishna, Power plant controls division, Indira Gandhi Centre for Atomic Research, Kalpakkam, India
The charge thus created in the ionization chamber by the above reactions, in tern ionizes the gas present in the chamber (normally Argon) and created charged ion pairs, which contribute to the following current pulse.

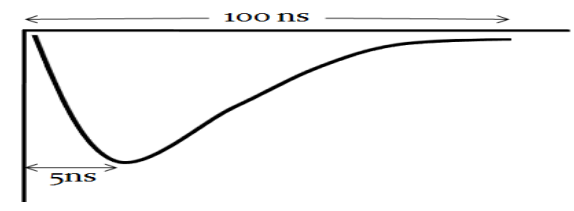

Fig. 1 - A single neutron pulse

\section{PUlSE DETAILS}

- The pulse width is variant. However, a width of 100 ns is a good estimate. The signal rise time varies from $5 \mathrm{~ns}$ to $20 \mathrm{~ns}$. The fall time varies from $50 \mathrm{~ns}$ to $120 \mathrm{~ns}$

- The amplitude of the signal is variant. It varies from $0.7 \mathrm{uA}$ to $1.3 \mathrm{uA}$

- The individual signals might overlap resulting in a single larger pulse.

- The signals will be affected by noise, which is also random in nature.

- The occurrence of signals follows a Poisson distribution.

- There is almost no overlap of signals for a frequency of less than $10^{4} \mathrm{~Hz}$.

- At very high frequencies, the pulse over lap fully to give average DC current.

- Due to the statistical nature of the signal, response time (tr) will be more at lower count rates and tr will be low at higher count rates.

- To overcome the above problem PCA technique had been devised.

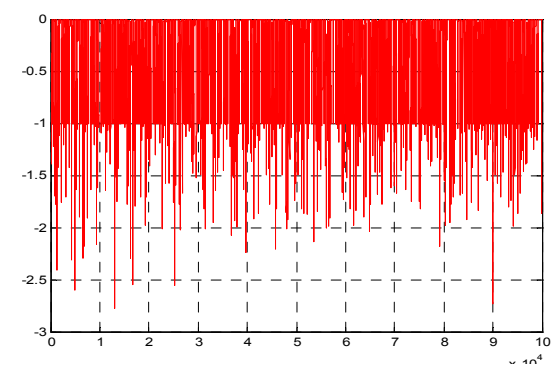

Fig.2 Overlapped pulse

Principal component analysis (PCA) has been called one of the most valuable results from applied linear algebra. PCA is used abundantly in all forms of analysis - from neuroscience to computer graphics because it is a simple, non-parametric method of extracting relevant information from confusing data sets. With minimal additional PCA 
provides a roadmap for how to reduce a complex data set to a lower dimension to reveal the sometimes hidden, simplified dynamics that often underlie it.

PCA is intimately related to the mathematical technique of Singular Value Decomposition (SVD), to determine the eigenvectors and Eigen values of the covariance matrices.

In general, each data sample is a vector in m-dimensional space, where $\mathrm{m}$ is the number of measurement types. Equivalently, different frequency signal are different dimensional vectors spanned by an orthonormal basis. All vectors in this space are a linear combination of this set of unit length basis vectors which is the regular pulse. What PCA does is that it finds another basis, which is a linear combination of the original basis that best re-expresses the data set.

\section{A. Assumptions for PCA}

- PCA makes one powerful assumption Linearity, which vastly simplifies the problem by restricting the set of potential bases, and formalizing the implicit assumption of continuity in a data set.

- PCA assumes P is an orthonormal matrix.

- PCA assumes the directions with the largest variances are the most "important" or in other words, most principal.

- The mean and the variance of data should be entirely described by a probability distribution. The only zeromean probability distribution that is fully described by the variance is the Gaussian distribution.

\section{B. Principal Component Analysis of Signal}

PCA is the simplest and most useful of the true eigenvector-based multivariate analyses, because its operation is to reveal the internal structure of data in an unbiased way. If a multivariate dataset is visualized as a set of coordinates in a high-dimensional data space ( 1 axis per variable), PCA supplies the user with a $2 \mathrm{D}$ picture, a shadow of this object when viewed from its most informative viewpoint.

PCA is equivalent to empirical orthogonal functions (EOF). In statistics and signal processing, the method of empirical orthogonal function (EOF) analysis is a decomposition of a signal or data set in terms of orthogonal basis functions which are determined from the data.

\section{Signal Processing and Algorithm}

Generate the random signal of Specified frequency for a sampling time of $10^{-4} \mathrm{sec}$ and sampling period of $10^{-9} \mathrm{sec}$. Add some high frequency noise to the signal keeping the Signal to Noise ratio high. Store it in the matrix Data of dimensions $\left(1 \times 10^{5}\right)$.

Organize the data set comprising of $10^{5}$ variables, take the transpose of the matrix Data converting it into a column vector of dimension $\left(10^{5} \times 1\right)$.

Find the empirical mean about the column dimension $m=$ $1 \ldots 10^{5}$. Place the calculated mean values into an empirical mean vector $u$ of dimensions $\left(10^{5} \times 1\right)$

Centering of the data by subtracting off the column mean is done to calculate the deviation from the mean and to minimize the mean square error of approximating the data. Store it in matrix $\mathrm{X}_{\mathbf{0}}$ of dimension $\left(10^{5} \times 1\right)$

Find the $\left(10^{5} \times 10^{5}\right)$ covariance matrix $\mathrm{C}$ from the outer dot product of $\mathrm{X}_{0}$ with itself.

Calculate the Eigen values and eigenvectors V, which Diagonalizes the Covariance matrix $\mathrm{C}$.

$$
V^{-1} C V=D
$$

This step will typically involve the use of a computerbased algorithm for computing eigenvectors and Eigen values. These algorithms are readily available as subcomponents of most matrix algebra systems.

In present case, $\mathrm{D}$ shall be a $(1 \times 1)$ matrix with the Eigen value of the covariance matrix for the particular random signal of specified frequency. Also the Principal Eigen vector $\mathrm{V}$ of covariance matrix $\mathrm{C}$ shall be a $(1 \times 1)$ matrix. The Eigen values and eigenvectors are ordered and paired.

Thus the Eigen value represents the degree of covariance of the signal with respect to itself. Hence with the increase of the frequency the degree of variation shall keep on increasing and that too linearly.

So calculate the Eigen value in the most principal direction and plot it with respect to the frequency to detect any random signal of arbitrary frequency.

The various stages of the processing are given in the following Fig.s

Fig. 3,4 give the random pulses at a frequency of 100 Khz.

Fig.5 give the appearance of the pulses when mean is subtracted.

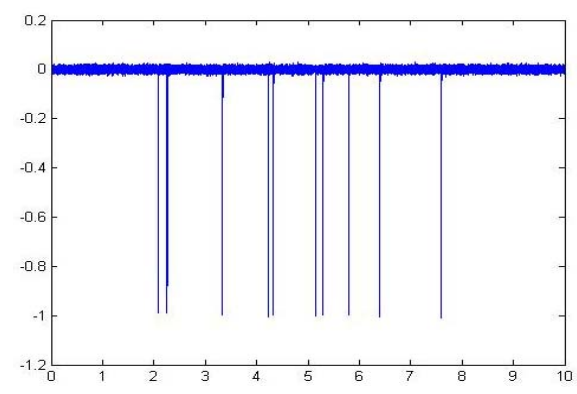

Fig. 3 - At frequency of $105 \mathrm{~Hz}$

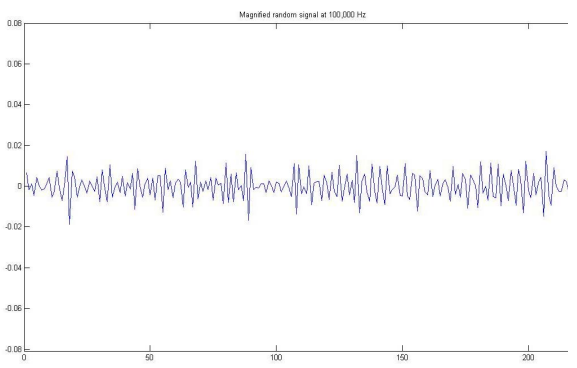

Fig. 4 - Magnified version of the Signal

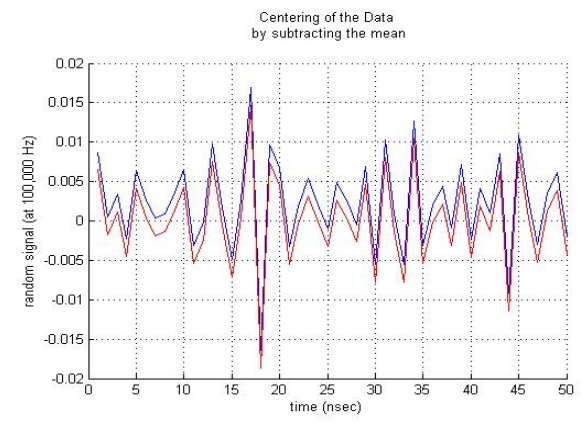

Fig. 5 - Centering of Data by subtracting the mean 


\section{Evaluating the Results of the OUtPut GENERATED}

1) Program executed from $10^{4}$ to $10^{5} \mathrm{~Hz}$ to generate the sample of 10 Eigen values about the Principal Axis for various frequencies.

The program was executed five times to check the validity of the result and also to study the effect of noise on the output. The result is very much coherent with rest of the analysis and thus reproducible and is given in the Fig. 6 given below.

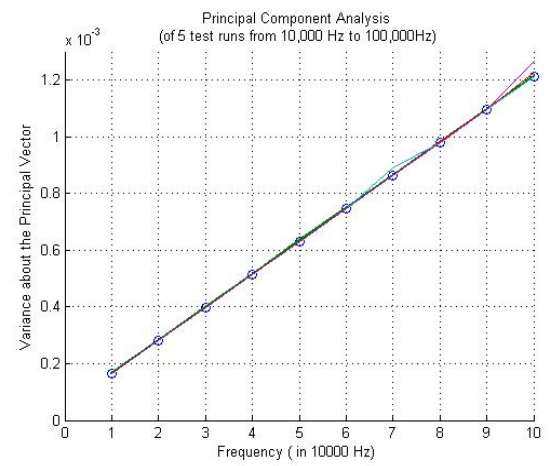

Fig. 6 - Result from 104 to $105 \mathrm{~Hz}$

2) Program executed from $10^{4}$ to $10^{6} \mathrm{~Hz}$ to generate the sample of 100 Eigen values about the Principal Axis for various frequencies. The program was executed five times to check the validity of the result. The result is given below in Fig.7.

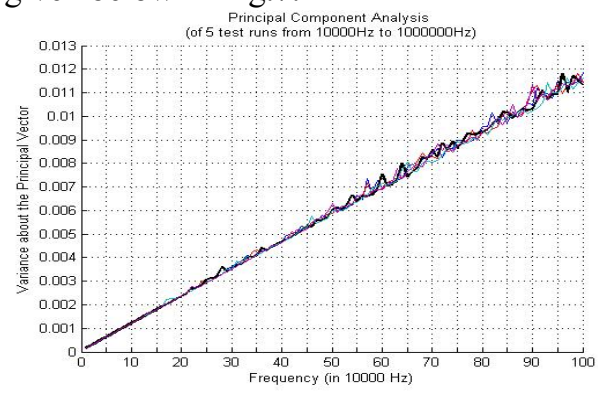

Fig. 7 - Result from 104 to $106 \mathrm{~Hz}$

Here interesting thing to notice is that as the signal reaches the value of about $50 \times 10^{4} \mathrm{~Hz}$ the linear graph for lower frequencies starts spiking whose range keeps on increasing with the frequency, this might result into error in final calculation at higher frequencies. It cannot be only because of the noise included as on average its effect is the same. The best suggestion is to further carry out the experiment till Best Fit Curve is achieved for various frequencies with respective error ranges.

3) Program executed form $10^{4}$ to $10^{7} \mathrm{~Hz}$ to generate the sample of 1000 Eigen values about the Principal Axis for various frequencies.

The program was executed five times to check the validity of the result and effect of noise on the output. The noise component in the Signal has more or less no effect till the Signal to Noise ratio is high, at least $82 \%$. The results are given below in the Fig.s 8-13.

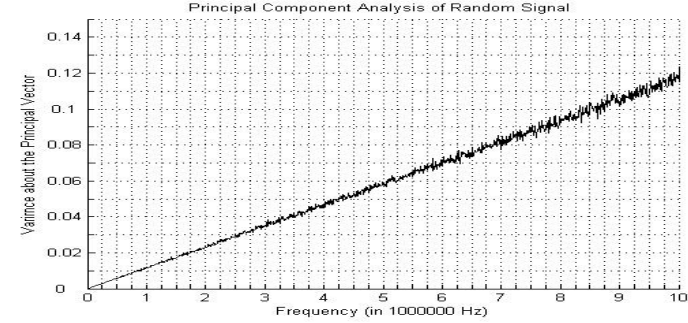

Fig. 8 - Test Run 1

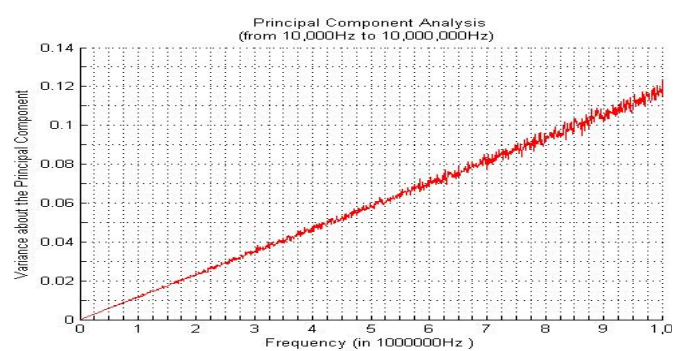

Fig. 9 - Test Run 2

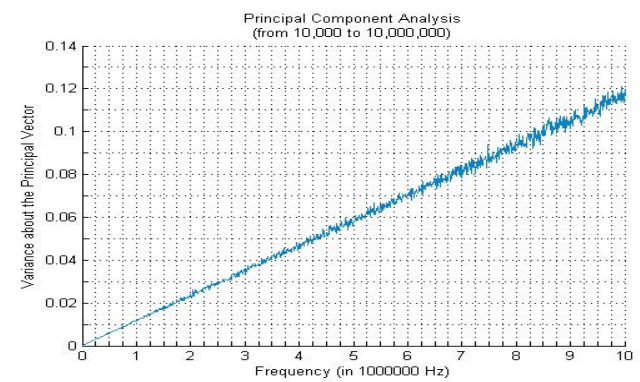

Fig. 10 - Test Run 3

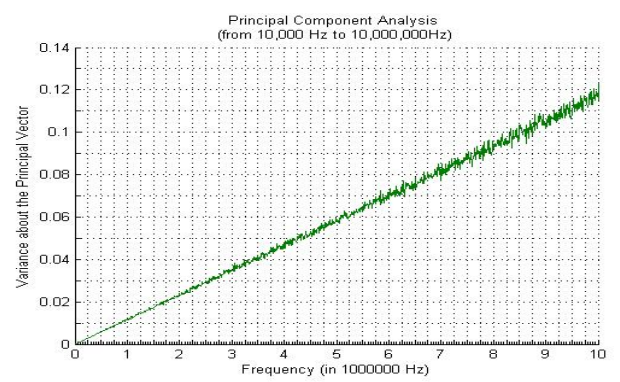

Fig. 11 - Test Run 4

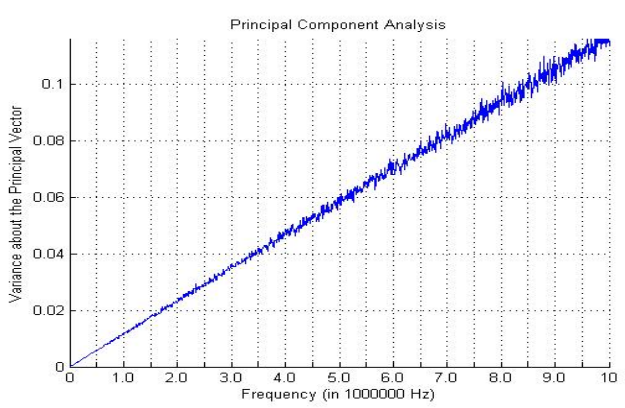

Fig. 12 - Test Run 5 


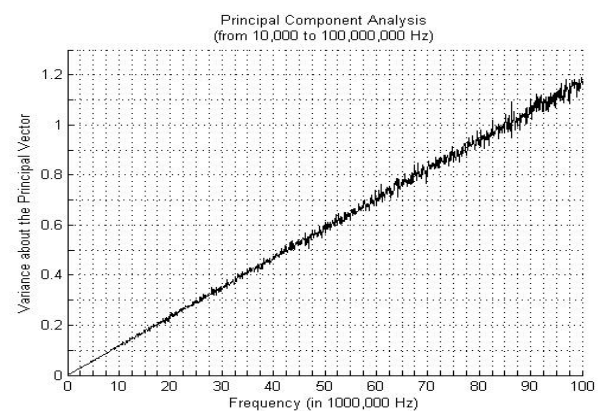

Fig. 13 - Test Run 6

4) Program executed form $10^{8}$ to $10^{9} \mathrm{~Hz}$ to generate the sample of 91 Eigen values about the Principal Axis for various frequencies. These are the compiled results for 3 test runs. The Eigen value increases drastically from 1 to 12 for the frequency ranging from $10^{8}$ to $10^{9} \mathrm{~Hz}$ showing that graph is nonlinearly increasing for higher frequencies. The result is given in the following Fig.s 14 and 15.

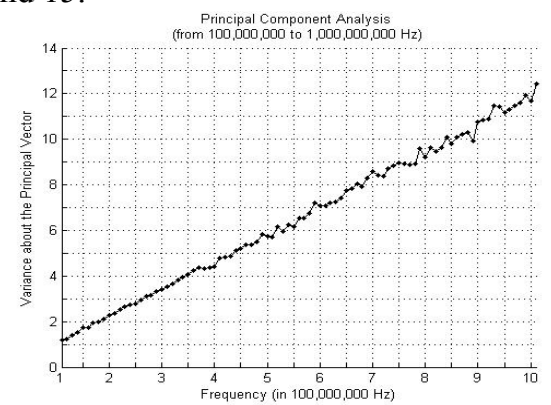

Fig. 14 - Result from $10^{8}$ to $10^{9} \mathrm{~Hz}$

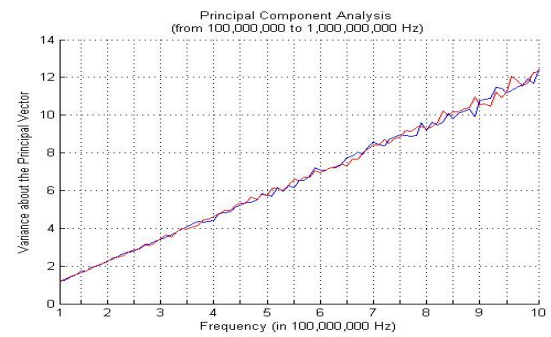

Fig. 15-Comparing the accuracy at $10^{9} \mathrm{~Hz}$
Also every time the code is executed the random signal is generated from the very beginning and then the noise is added so these results are pretty accurate, although the accuracy can be further improved.

\section{CONCLUSION}

Conventional counting technique can't be applied for counting neutron pulses beyond $1 \mathrm{Mhz}$, due to randomness and pulse overlap. Hence special method viz. PCA is devised. This technique could establish linear correlation between certain PCA parameters with frequency of pulses.

\section{DISCUSSION}

In the present technique, the degree of variation is calculated so it kills the randomness. But the disadvantage is that at higher frequencies the curves gives spikes. And also the error range increases at higher frequencies, and is needed to be calculated through curve fitting. Hence special techniques such as neural networks can be used to cover the anomalies in the curves at higher frequency. In case of any discrepancy, artificial neural networks can perform input output mapping pretty accurately.

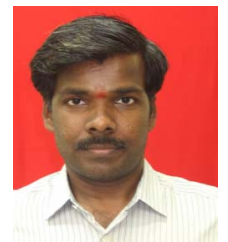

Metta Sivaramakrishna was born in Secunderabad, Andhra Pradesh, India in 1970. He obtained his B.Tech degree in Electronics and Communication from the Institute Jawaharlal Nehru Technological University, Hyderabad, India. He joined Power Plant controls Division of Indira Gandhi Centre for Atomic Research, Kalpakkam, India in 1992. He is specialist in the field of analog and digital electronics and embedded systems. He has also experience in circuit simulation and numerical simulation techniques. Presently he is doing research in advanced signal processing techniques of neutron detectors for fast breeder reactors. 